THE CHANGING FACE OF CLINICAL TRIALS

Jeffrey M. Drazen, M.D., David P. Harrington, Ph.D., John J.V. McMurray, M.D., James H. Ware, Ph.D., and Janet Woodcock, M.D., Editors

\title{
Lessons in Uncertainty and Humility - Clinical Trials Involving Hypertension
}

\author{
Marc A. Pfeffer, M.D., Ph.D., and John J.V. McMurray, M.D.
}

\begin{abstract}
From the Cardiovascular Division, Brigham and Women's Hospital, Harvard Medical School, Boston (M.A.P.); and the British Heart Failure Cardiovascular Research Centre, University of Glasgow, Glasgow, United Kingdom (J.J.V.M.). Address reprint requests to Dr. Pfeffer at the Cardiovascular Division, Brigham and Women's Hospital, 75 Francis St., Boston, MA 02115, or atmpfeffer@rics.bwh.harvard.edu.

N EngIJ Med 2016;375:1756-66. DOI: 10.1056/NEJMra1510067

Copyright (@) 2016 Massachusetts Medical Society.
\end{abstract}

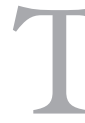
HE CENTRAL TENET OF CLINICAL RESEARCH IN HUMANS IS THAT PARticipation is informed and voluntary. Participation is considered to be informed only when there are properly scrutinized protocols and when participants are fully informed of the known risks, discomforts, and inconveniences related to their involvement. ${ }^{1}$ Across the many types of clinical investigation, the randomized, controlled trial is the key scientific tool used to measure the efficacy and adverse effects of a diagnostic or therapeutic approach. ${ }^{2}$ Randomization, the critical element used to reduce bias, not uncommonly creates ethical quandaries, since the treating physician and the patient, by agreeing to participate in the trial, forgo their choice of the intervention being tested, leaving this to chance. ${ }^{3}$ For those designing the trial, making the decision as to whether the comparator treatment should be an inert substance - that is, a placebo - or an active treatment can be difficult. For those making the decision as to whether to participate, it is important that they fully understand the medical question being explored and the role they will play in helping to obtain an answer.

Clinical trials do not occur in a vacuum. Before a major clinical-outcome trial is started, there is often substantial information available about the intervention from smaller, hypothesis-generating trials that are usually intended to examine the effects of the intervention on less definitive outcomes, such as a biomarker or a surrogate end point that is believed to correlate with changes in clinical events. In some cases observational data may be available, and in other instances previous clinical-outcome trials have established the effectiveness of an agent but have done so in a different patient population. Sometimes this existing information engenders divergent and even polarized opinions. In that case, a randomized trial of high quality should be welcomed by all parties as the best means to resolve the controversy. Even so, strong preferences may undermine support for the randomization process, affecting the veracity of the trial by injecting bias into the selection of patients. In other instances, a solid consensus coalesces around what are perceived to be the best existing data. When the intervention in question is already generally part of clinical practice, many health professionals find comfort in its use and view a randomized trial as unnecessary or even unethical, since some patients would not receive the therapy that they would have received in the absence of the trial. In this article, we use examples from cardiovascular medicine to discuss the effects of the existing and evolving information on trial design with regard to the choice of placebo or active comparator agents and to the definition of the population of patients in the trial.

Regardless of whether the prestudy perception is one of clinical equipoise or involves a range of uncertainty, if a randomized trial is to be initiated and con- 
ducted, the fundamental question it addresses must be worthy of the major collective efforts required of patients, investigators, and sponsors. ${ }^{4}$ It is important to note that regardless of the level of confidence in the prestudy information, a randomized, controlled trial remains a scientific experiment. Properly worded consent forms should not lead a participant to expect any unproven benefits.

Notwithstanding the careful planning of the experts who design the trial, the scrutiny of regulatory agencies, and the commitment of resources from sponsors to demonstrate proposed theoretical benefits, many researchers conclude that the intervention they had hoped would offer a diagnostic or therapeutic advance is no better than the existing options. ${ }^{5,6}$ The effectiveness of an intervention is best evaluated in randomized trials in which well-defined clinical events are the primary outcome.

In the absence of clinical-outcome data that provide definitive direction, surrogate measures are often used to direct therapeutic decisions. Consistent linkage from reliable observational data between a commonly used biologic measurement and prognosis can understandably lead to a perceived association that changes in that biomarker can be reasonably assumed to cause directionally similar alterations in clinical outcomes. For many of these biologic measures, such as levels of high-density lipoprotein cholesterol or hemoglobin, the density of ventricular arrhythmias, and left ventricular ejection fraction, multiple observational studies are available that show such quantitative associations with cardiovascular risk. ${ }^{7}$ The finding of a quantitative relationship between the degree of deviation of the measured marker from normality and adverse outcomes has on occasion fostered the reasonable expectation for both drug discovery and clinical practice that therapies that restore the abnormal measurement toward normal would have a favorable outcome.

In cardiovascular medicine, this comfort zone was shattered by the results of the Cardiac Arrhythmia Suppression Trial, which showed that despite the clear association between the degree of ventricular ectopy and risk of death, antiarrhythmic therapy, which was effective in suppressing ventricular premature beats, increased rather than decreased mortality. ${ }^{8}$ Before this placebo-controlled trial was conducted, a major emphasis was placed on detecting patients with asymptomatic ventricular arrhythmias in order to initiate antiarrhythmic agents that would provide the assumed benefits. The totally unexpected result - that treating these abnormal rhythms with antiarrhythmic agents was associated with increased mortality - painfully disclosed the false sense of security of what was then a widely adopted clinical practice. The prompt translation of these findings into reductions in the use of these unsafe therapies improved public health and created additional uncertainties. These surprising findings also raised questions about other cardiovascular therapies whose use had became widespread after regulatory evaluations were satisfied, predominantly on the basis of alterations of surrogate measures rather than clinical events.

EARLY DATA ON BLOOD PRESSURE AND OUTCOMES

Our story is about the treatment of hypertension and the finding in multiple trials that the normalization of blood pressure has been consistently accompanied by beneficial clinical outcomes (Fig. 1). Elevations in systemic arterial pressure were identified in early pioneering epidemiologic studies (e.g., the Framingham Heart Study and the Multiple Risk Factor Intervention Trial [MRFIT]) as being tightly coupled to a heightened risk of illness and death from cardiovascular disease. ${ }^{9,10}$ A historical review of some of the predominantly government-sponsored randomized trials that were designed to ascertain whether and when long-term antihypertensive therapy lowers these risks illustrates several of the ethical issues that influence the comparators and the patient populations chosen for study as new information is generated.

One of the early randomized, placebo-controlled, clinical-outcome trials in cardiovascular medicine, commencing in 1963, was conducted by the Veterans Administration (VA) Cooperative Study Group on Antihypertensive Agents. In its trial involving 143 men whose diastolic blood pressure was between 115 and $129 \mathrm{~mm} \mathrm{Hg}$, the group found that the use of a combination of three antihypertensive agents resulted in fewer cardiovascular events than the use of placebo. ${ }^{11}$ Since the risks and the potential benefits of using pharmacologic agents to reduce blood pres- 


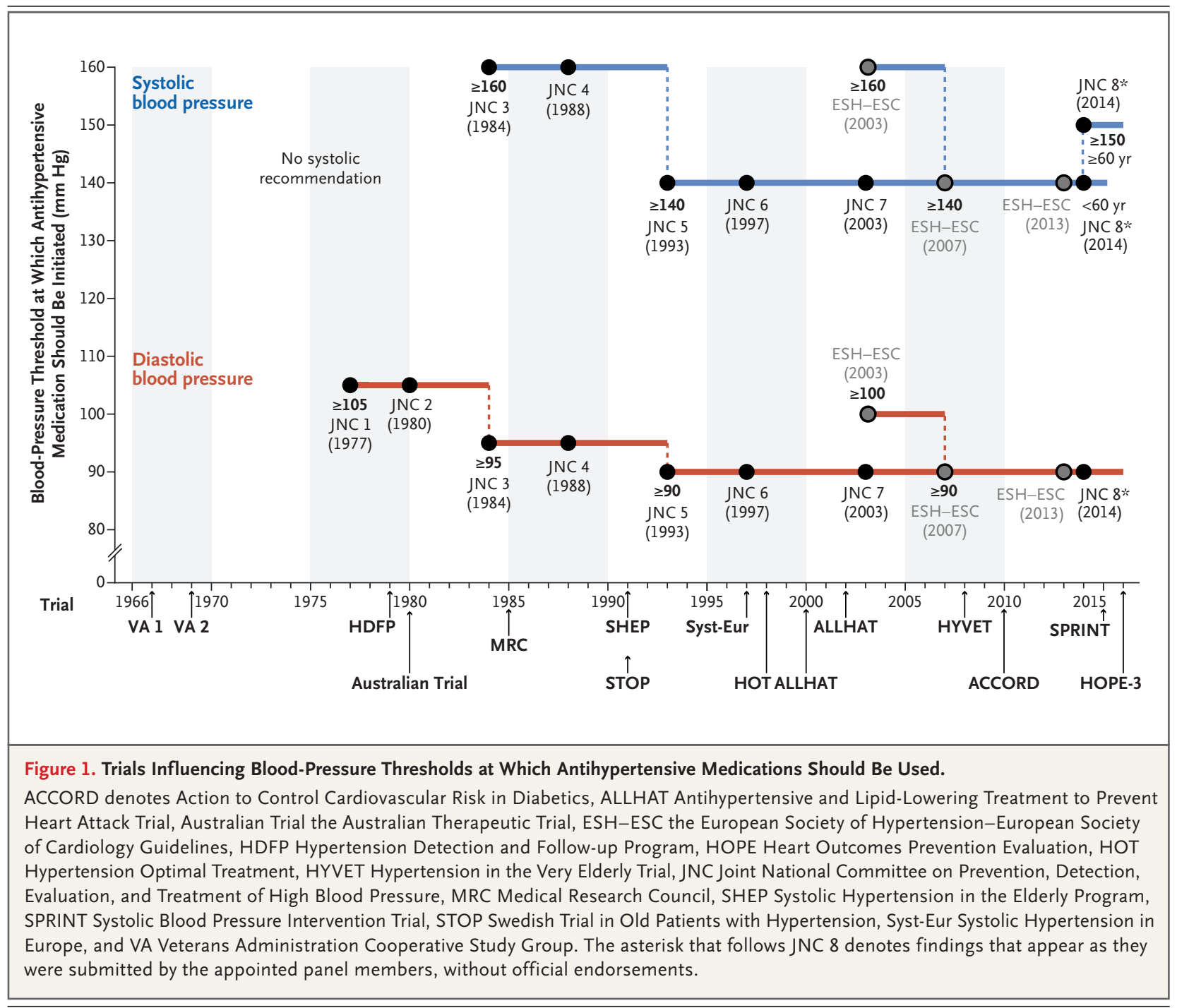

sure were unknown, placebo was unquestionably the correct comparator. The same group concurrently conducted another trial of 380 men whose diastolic pressures were between 90 and $114 \mathrm{~mm} \mathrm{Hg}{ }^{12}$ The lower diastolic pressure of the participants in this trial made placebo the appropriate control for this previously unstudied population. The combination of the participants' lower diastolic pressure and the fact that this population had not been studied previously also made placebo the appropriate control in this trial. The findings of each of these placebo-controlled trials, which showed that the adverse cardiovascular consequences of elevated blood pressure could be reduced by the administration of antihypertensive medications, are now considered to be foundational evidence for the concept of pre- ventive medicine. However, at the time, the clinical importance of treating an asymptomatic person with the available therapies for lowering blood pressure was not readily accepted by the medical community.

\section{RECOGNITION OF THE DANGERS OF HYPERTENSION}

The National High Blood Pressure Education Program was established in 1972 by philanthropist Mary Lasker and then-Secretary of Health, Education, and Welfare Elliot Richardson with the objective of alerting physicians and the public to the risks of hypertension. ${ }^{13}$ This governmentfunded educational program used phrases such as "the silent killer" to heighten awareness of 
the dangers of elevated blood pressure and to encourage the use of antihypertensive therapy in patients with hypertension. The reluctance of the medical community to accept the findings of the two VA trials was reflected in the design of subsequent major trials focused on hypertension.

The investigators conducting the Hypertension Detection and Follow-up Program (HDFP) that was sponsored by National Institutes of Health designed their trial to determine whether improved blood-pressure control could prolong survival. The trial included nearly 11,000 people, and enrollment began in 1974. Given that study entry required a diastolic blood pressure of at least $90 \mathrm{~mm} \mathrm{Hg}$ (with more than 1000 participants having a diastolic pressure of more than $115 \mathrm{~mm} \mathrm{Hg}$ at study entry), the HDFP was in some respects an effort to confirm the results of the VA trials while also expanding the population covered to include women and younger people. Despite these population differences, the designers of the HDFP "agreed that the results of the Veterans trials made it inappropriate to use placebo controls." ${ }^{14}$ This clear statement and early shift from placebo to an active comparator indicated that the investigators believed that it was unethical to withhold the opportunity for antihypertensive therapy from participants during a long-term clinical trial. However, the tension between clinical investigators and community practitioners with regard to the acceptance of the existing data was reflected in the trial design and the differential use of antihypertensive drugs between groups.

In this 5 -year HDFP, a total of 10,940 volunteers ( $46 \%$ of whom were women) were randomly assigned to receive either stepped care (SC), in which antihypertensive therapy was provided at specialized sites and doses increased to achieve and maintain specific blood-pressure goals, or referred care (RC), in which participants were referred to their usual sources of care. The design was based on an inherent assumption that despite the absence of placebo, the ambivalence of the medical community toward antihypertensive drugs would result in fewer prescriptions in the RC group than in the SC group. Although a considerable number of patients in each group remained untreated, the percentage was more than twice as high in the RC group at year 2, with $18.5 \%$ of the SC group and $41.7 \%$ of the RC group remaining untreated. The goal of attain- ing a diastolic pressure of less than $90 \mathrm{~mm} \mathrm{Hg}$ was achieved in $56.7 \%$ of the patients in the SC group and in only $33.7 \%$ of the patients in the RC group. Even this difference in the intensity of blood-pressure treatment was associated with a statistically and clinically significant difference in mortality, which was $17 \%$ lower in the SC group than in the RC group. ${ }^{14}$

The response of the international medical community to the results of the two relatively small initial VA studies was even more restrained than that in the United States. Near the time at which the HDFP was being conducted in the United States, three trials related to hypertension control were being performed outside the United States. In each of these trials, placebo was selected as the comparator (Table 1). Entry into the European Working Party Trial ${ }^{16}$ and the Medical Research Council Trial (conducted in the United Kingdom $)^{17}$ required a diastolic pressure of $90 \mathrm{~mm} \mathrm{Hg}$ or higher, and entry into the Australian Therapeutic Trial ${ }^{15}$ required a diastolic pressure of $95 \mathrm{~mm} \mathrm{Hg}$ or higher. The use of placebo in all three trials, which commenced in the 1970 s, was a clear indication that the researchers' level of uncertainty with regard to the need to treat people with this level of blood pressure was higher than it was for the U.S. investigators in the HDFP. Nonetheless, hypertension knows no borders, and all three trials showed that fewer cardiovascular events occurred among patients with better blood-pressure control. With these findings, the importance of lowering blood pressure was solidified (Table 1).

The design of the Hypertension Optimal Treatment (HOT) trial, conducted in the mid-1990s, showed that the international community of researchers who were investigating hypertension had accepted the proposition that in patients with elevated diastolic blood pressure, the reduction of that pressure with antihypertensive drugs improved cardiovascular outcomes. ${ }^{21}$ In the HOT trial, the diastolic pressure of participants was between 100 and $115 \mathrm{~mm} \mathrm{Hg}$, and all received active antihypertensive therapy. Participants were randomly assigned to one of three groups in which the targeted diastolic blood pressures were less than 90 , less than or equal to 85 , or less than or equal to $80 \mathrm{~mm} \mathrm{Hg}$. Although no significant differences in the rates of major cardiovascular events were observed across the three levels of treatment intensity, this trial, which included 


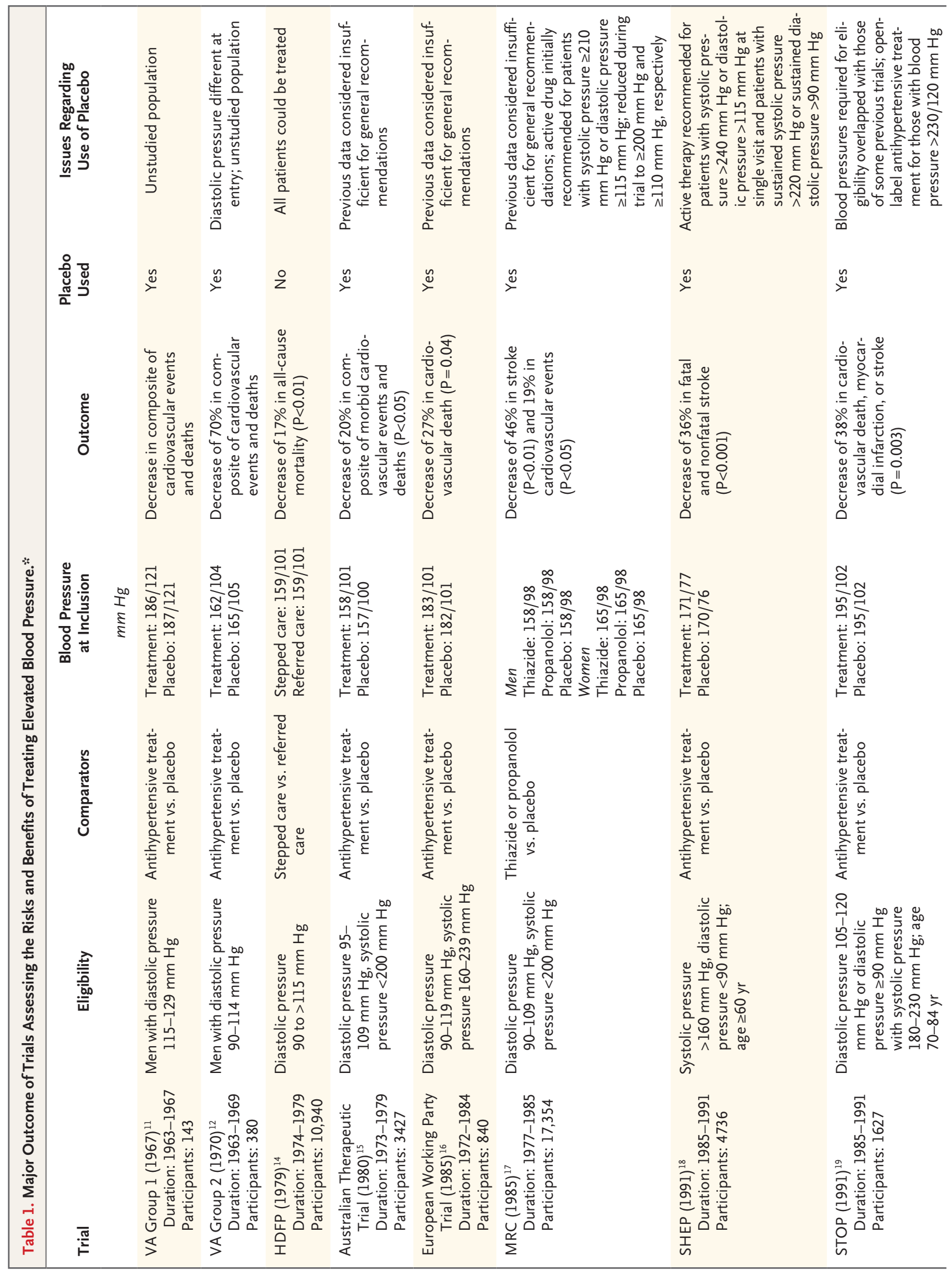




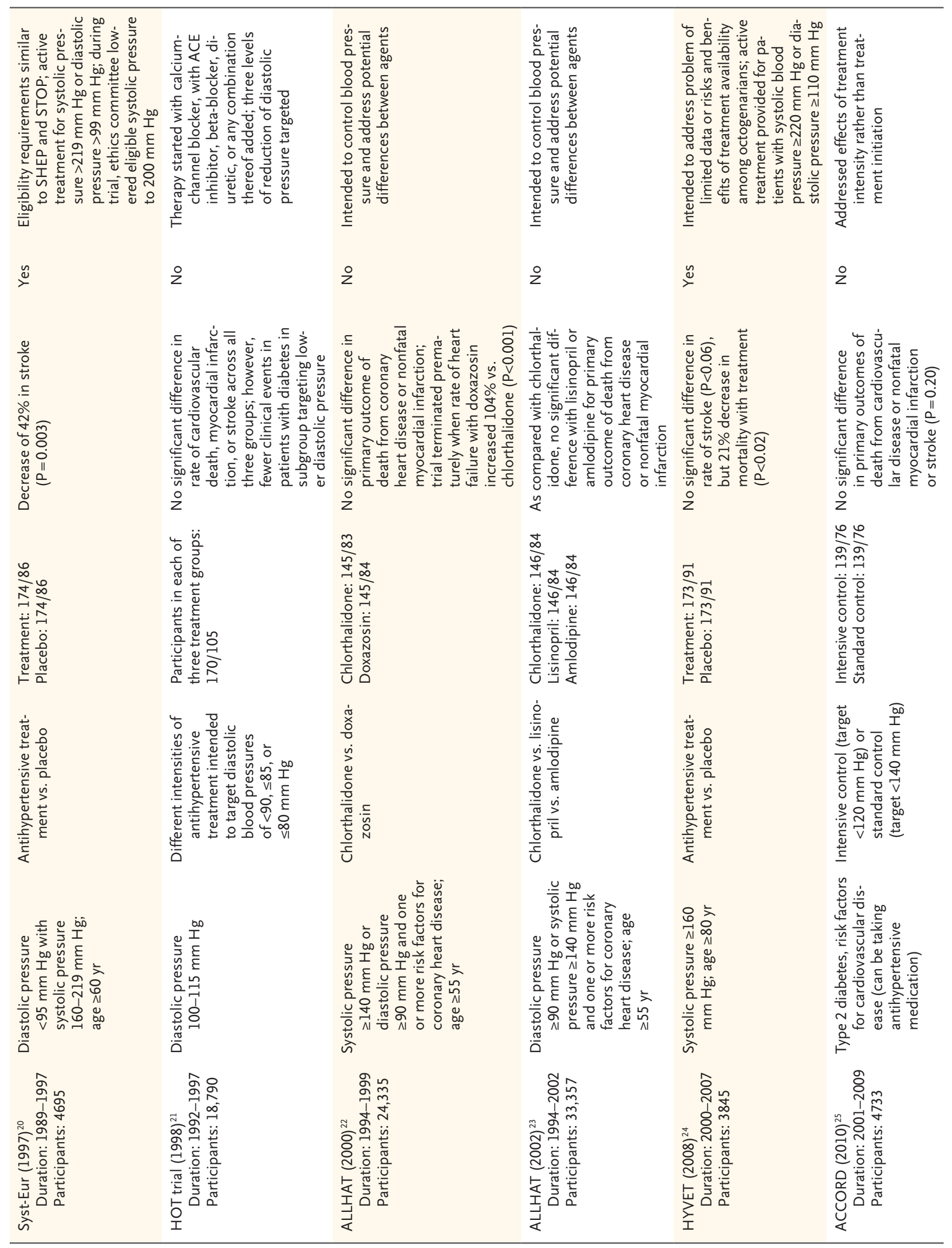




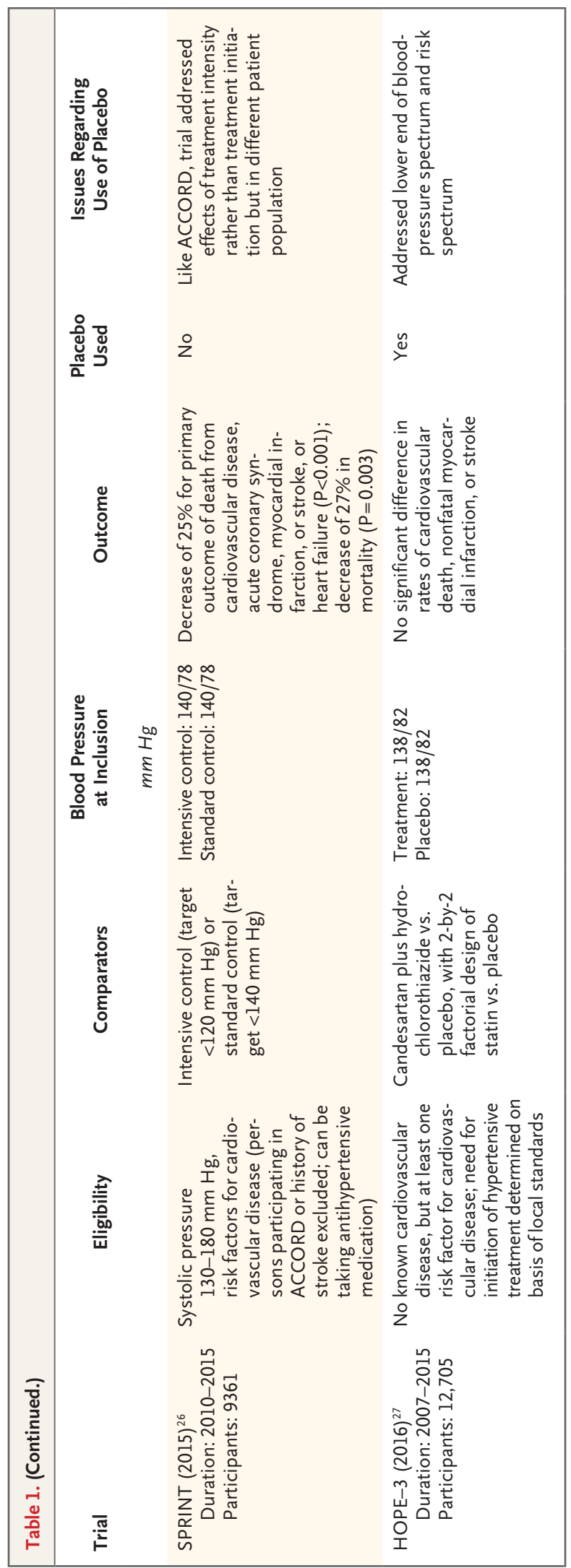

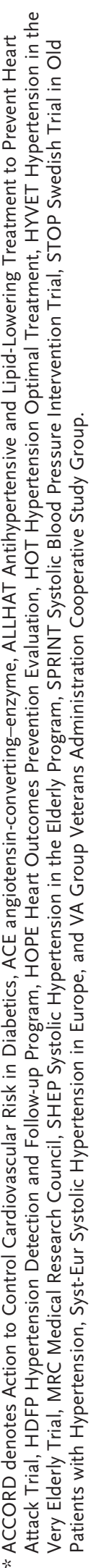

18,790 participants, made a clear statement that elevated diastolic pressure should be lowered to at least $90 \mathrm{~mm} \mathrm{Hg}$.

SHIFT TO SYSTOLIC PRESSURE

The Systolic Hypertension in the Elderly Program (SHEP), which was conducted from 1985 through 1991, involved 4736 patients, was one of the first trials to address the use of systolic blood pressure as a guide for initiating antihypertensive treatment. In order to participate, a patient had to have a systolic blood pressure between 160 and $219 \mathrm{~mm} \mathrm{Hg}$ and a diastolic pressure of less than $90 \mathrm{~mm} \mathrm{Hg}$ (average blood pressure, $170 / 77 \mathrm{~mm} \mathrm{Hg}) .^{18}$ Although these levels of systolic pressure are very high by today's standards, the benefits and risks of antihypertensive therapy in this generally older population were unknown at the time the trial was conducted. Although placebo was used as the control, the trial included an "escape" clause that allowed for the initiation of open-label therapy for patients whose systolic blood pressure was higher than $220 \mathrm{~mm} \mathrm{Hg}$. At year 2, a total of $6.7 \%$ of the active-treatment group and $70.9 \%$ of the placebo group were not being treated, and the betweengroup difference in systolic pressure was approximately $13 \mathrm{~mm} \mathrm{Hg}$. The active-treatment group had a rate of stroke that was 36\% lower than that in the placebo group and a composite rate of nonfatal myocardial infarction, death from coronary heart disease, or heart failure that was $33 \%$ lower.

The European Working Party on High Blood Pressure in the Elderly investigated isolated systolic hypertension in the Systolic Hypertension in Europe (Syst-Eur) trial. ${ }^{20}$ The entry criterion for this randomized, placebo-controlled trial was a systolic blood pressure of 160 to $219 \mathrm{~mm} \mathrm{Hg}$, with a diastolic pressure of less than $95 \mathrm{~mm} \mathrm{Hg}$. The Syst-Eur trial, which was conducted from 1989 through 1997, was continued after the results of SHEP were known "because of remaining uncertainties about the treatment of isolated systolic hypertension in the elderly." ${ }^{20}$ The trial was stopped after an interim analysis showed that the incidence of the primary end point (stroke) was $42 \%$ lower in the active-treatment group than in the placebo group $(\mathrm{P}=0.003)$. The incidence of heart failure and myocardial infarction was $25 \%$ lower in the active-treatment group. 
The Swedish Trial in Old Patients with Hypertension (STOP-Hypertension) was also conducted during this period (1985-1991) and also used placebo rather than active treatment in the comparator group. ${ }^{19}$ On entry into the trial, participants had to have a diastolic blood pressure between 105 and $120 \mathrm{~mm} \mathrm{Hg}$ (irrespective of systolic pressure) or both a diastolic pressure of at least $90 \mathrm{~mm} \mathrm{Hg}$ and a systolic pressure between 180 and $230 \mathrm{~mm} \mathrm{Hg}$. The diastolic criteria overlapped with those for participation in previous trials, as the investigators acknowledged in their stated objective: "to confirm the value of antihypertensive treatment in people age 70-74 years and to expand the database up to the age of 84 years." The blood-pressure levels at year 2 of the study were $188 / 97 \mathrm{~mm} \mathrm{Hg}$ in the placebo group and $166 / 87 \mathrm{~mm} \mathrm{Hg}$ in the treatment group. The rate of cardiovascular death, myocardial infarction, or stroke was $38 \%$ lower in the activetreatment group (Table 1).

These trials from the 1980s and early 1990s generated the data needed to develop a systolic blood-pressure target for the initiation of antihypertensive therapy. The cumulative evidence provided the basis for the recommendations initially made in the 1993 report of the Joint National Committee on Prevention, Detection, Evaluation, and Treatment of High Blood Pressure (Fig. 1) to start long-term antihypertensive therapy at a systolic pressure of $140 \mathrm{~mm} \mathrm{Hg}$ or higher or a diastolic pressure of $90 \mathrm{~mm} \mathrm{Hg}$ or higher.

EMERGENCE OF ETHICAL ISSUES AS KNOWLEDGE ACCUMULATES

The ethical boundaries of finding sufficient uncertainty for the use of placebo in patients with hypertension were probed more recently in the Hypertension in the Very Elderly Trial (HYVET), which was conducted from 2000 through 2007. In this major outcome trial, active antihypertensive therapy was compared with placebo in patients 80 years of age or older who had a systolic pressure of $160 \mathrm{~mm} \mathrm{Hg}$ or higher. ${ }^{24}$ The rationale was that minimal data on the effectiveness or safety of antihypertensive therapy were available in this population. Although the rate of the primary end point of stroke was not significantly lower than the rate with placebo, the $21 \%$ lower rate of death $(\mathrm{P}<0.02)$ in the active antihyperten- sive-therapy group confirmed the importance of lowering blood pressure regardless of age and closed the chapter on the use of placebo in controlled trials addressing this level of hypertension (Table 1).

In the Heart Outcomes Prevention Evaluation (HOPE) -3 trial, ${ }^{27}$ the results of which were reported in spring 2016, the investigators used placebo as the comparator with active combination antihypertensive therapy to evaluate the risks and benefits of blood-pressure control in persons whom investigators believed did not have a clear current indication for the active therapies being evaluated. Participants could enter the trial after blood-pressure control was attained with lifestyle interventions or with drugs other than an angiotensin-receptor-blocker, an angiotensin-converting-enzyme inhibitor, or a thiazide diuretic. In effect, this design, which permitted some participants in the control group to receive antihypertensive therapy, was a hybrid between no treatment and add-on therapy, since at baseline $22 \%$ of patients were already receiving treatment intended to lower blood pressure. With the mean blood pressure at baseline at $138 / 82 \mathrm{~mm} \mathrm{Hg}$, some participants had a bloodpressure level that was above the existing treatment targets and the targets being introduced at the time the trial started (Fig. 1).

The trial results were neutral, with no significant difference reported between placebo and active therapy for the composite rate of cardiovascular events. However, in a subgroup analysis of the participants with the highest systolic pressure at trial entry $(>143.5 \mathrm{~mm} \mathrm{Hg}$ ), those who received combination antihypertensive therapy had fewer cardiovascular events than those receiving placebo. What made this modern placebo-controlled trial distinctive was the fact that it explored the lower limits at which antihypertensive therapy should be provided by offering treatment to participants who were not considered to need either initiation of such treatment or any additional treatment - an understudied population with an annual risk of major cardiovascular events of approximately $1 \%$. The trial also had a 2-by-2 factorial design in which a statin was provided for the purpose of preventing cardiovascular events without the use of lipid or blood-pressure targets or monitoring.

As the data on the benefits of antihypertensive therapy have increased, ethical standards in 
this field have continued to evolve. ${ }^{28}$ Generally, institutional review boards will permit only shortterm withholding (approximately 2 to 4 weeks) of antihypertensive medications (within a nonsevere blood-pressure range) to establish baseline blood pressure. Most current trials assessing the effects of lowering blood pressure compare the effects of a new therapy with those of an established comparator rather than placebo. ${ }^{23,28-31}$ The comparisons between specific antihypertensive agents are often made at doses that achieve similar degrees of blood-pressure control. Although some large clinical-outcome trials that compare specific antihypertensive agents have been conducted to identify possible benefits of treatment other than that of lowering blood pressure,,$^{23,29,31}$ for the most part reductions in the occurrence of major cardiovascular events are more closely related to the extent to which blood pressure is lowered than to the class of agent used. ${ }^{32}$ One of the largest trials in which active agents were used as comparators was the Antihypertensive and Lipid-Lowering Treatment to Prevent Heart Attack Trial (ALLHAT) sponsored by the National Heart, Lung, and Blood Institute (NHLBI), in which a diuretic was compared with three other classes of antihypertensive drug. ${ }^{23}$ In this trial, which was conducted from 1994 through 2002, the finding that the risk of the development of heart failure was twice as high with doxazosin as it was with chlorthalidone provided the research community with an important lesson in humility. ${ }^{22}$

\section{BEYOND PLACEBO}

The clinical-outcome trials involving hypertension that were conducted in the past decade addressed the level at which antihypertensive treatment should be initiated and at what intensity; these trials did not use a placebo. Since the epidemiologic data relating blood pressure and cardiovascular risk do not show a clear threshold (i.e., a level below which risk does not appear to diminish), ${ }^{9,10}$ there was a widely held belief that the use of antihypertensive drugs to bring blood pressure below the operational definition of hypertension would further improve prognosis. For patients with type 2 diabetes, this belief was supported by a subgroup analysis from the HOT trial that showed that among the 1501 patients in the trial who had diabetes, there was a gradation in the observed rate of cardiovascular events across the three groups that had met the target diastolic pressure. ${ }^{21}$ This finding led to the recommendation of an even lower target blood pressure in these patients. ${ }^{28}$ However, it was acknowledged that this target was based on subgroup data.

There was sufficient uncertainty regarding the recommendation that one aspect of the NHLBIsponsored Action to Control Cardiovascular Risk in Diabetics (ACCORD) trial was specifically designed to address this question..$^{25}$ In ACCORD, an open-label trial that commenced in 2001, patients were randomly assigned to receive either intensive antihypertensive therapy targeting systolic blood pressures of less than $120 \mathrm{~mm} \mathrm{Hg}$ or standard therapy targeting systolic blood pressures of less than $140 \mathrm{~mm} \mathrm{Hg}$. The group receiving standard treatment was to have at least the currently recommended level of blood-pressure control, and the focus was on between-group differences in blood-pressure levels, not on the specific antihypertensive agents used. The group receiving intensive treatment was in effect providing the unknown relative efficacy and safety data related to the use of a greater number of antihypertensive medications intended to lower blood pressure to levels not previously studied in major clinical-outcome trials.

Despite achieving an impressive average difference of $14 \mathrm{~mm} \mathrm{Hg}$ in systolic pressure, the primary composite outcome of time to cardiovascular death, myocardial infarction, or stroke was not lower in the group receiving intensive therapy than in the group receiving standard therapy. The trial also showed that there was a downside to the strategy of striving for greater and greater reductions in blood pressure, since a higher number of serious adverse events (i.e., events that were life-threatening, caused permanent disability, or necessitated hospitalization) that were attributed to antihypertensive medications were reported in the group receiving intensive treatment. The results of the ACCORD trial did not align with expert consensus, ${ }^{34}$ which provided another lesson in humility from a wellconducted outcome trial that improved practice and defied expert opinion.

The even more recent NHLBI-sponsored Systolic Blood Pressure Intervention Trial (SPRINT) ${ }^{26}$ 
the results of which were published in 2015, addressed the same question in a population with a high level of cardiovascular risk. However, in light of the neutral findings related to target blood pressures from the ACCORD trial and the Secondary Prevention of Small Subcortical Strokes trial, ${ }^{33}$ the SPRINT investigators excluded patients with diabetes or a history of stroke. Again, the design was a randomized, controlled trial in which patients used open-label antihypertensive agents to a target systolic pressure of either less than 120 or less than $140 \mathrm{~mm} \mathrm{Hg}$. Although in the ACCORD trial the group receiving the more intensive approach to lowering systolic blood pressure did not have a benefit-risk profile that justified this approach in persons with diabetes, the SPRINT investigators felt justified in testing the same hypothesis in patients with elevated blood pressure who were not known to have diabetes. On the heels of the ACCORD trial, the early termination of the SPRINT trial for reasons of efficacy came as a surprise. The data showed that more intensive lowering of blood pressure, below the currently recommended level, significantly reduced rates of cardiovascular end points and the risk of death. ${ }^{26}$ Once scrutinized, it seems likely that this new information will once again change current authoritative recommendations for standard practice in an effort to improve public health (Fig. 1). ${ }^{34}$

\section{LESSONS LEARNED}

The privilege of conducting human research requires investigators to minimize the risks to participants and to inform participants of these risks by providing them with the most accurate current information available. Randomized trials should be conducted within an ethical framework that allows all participants access to the best possible care (as determined regionally) and to an experimental comparator about which the preliminary information is sufficient to suggest a degree of efficacy that justifies the conduct of a clinical trial (given the inherent uncertainty involved). As new information is generated and the degree of uncertainty diminished, these developments should be reflected in the selection of proper comparators. The history of randomized, controlled trials involving hypertension shows that the level of uncertainty or, conversely, the interpretation of the robustness of the currently available data can vary among physicians within a country and across geographic regions. A review of this history underscores the sanctity of the central tenet of the consent process: properly informing the voluntary participants of relevant information at the time of enrollment so that they can make a truly informed decision.

Disclosure forms provided by the authors are available with the full text of this article at NEJM.org.
REFERENCES

1. Grady C. Enduring and emerging challenges of informed consent. $\mathrm{N}$ Engl J Med 2015;372:855-62.

2. Califf RM, DeMets DL. Principles from clinical trials relevant to clinical practice. Circulation 2002;106:1015-21.

3. Chalmers TC, Celano P, Sacks HS, Smith H Jr. Bias in treatment assignment in controlled clinical trials. N Engl J Med 1983;309:1358-61.

4. Eisenstein EL, Lemons PW II, Tardiff BE, Schulman KA, Jolly MK, Califf RM. Reducing the costs of phase III cardiovascular clinical trials. Am Heart J 2005;149: 482-8.

5. Ridker PM, Torres J. Reported outcomes in major cardiovascular clinical trials funded by for-profit and not-forprofit organizations: 2000-2005. JAMA 2006;295:2270-4.

6. Gordon D, Taddei-Peters W, Mascette A, Antman M, Kaufmann PG, Lauer MS. Publication of trials funded by the National Heart, Lung, and Blood Institute. N Engl J Med 2013;369:1926-34.
7. Fleming TR, DeMets DL. Surrogate end points in clinical trials: are we being misled? Ann Intern Med 1996;125:605-13.

8. Echt DS, Liebson PR, Mitchell LB, et al. Mortality and morbidity in patients receiving encainide, flecainide, or placebo: the Cardiac Arrhythmia Suppression Trial. N Engl J Med 1991;324:781-8.

9. Kannel WB, Dawber TR, Kagan A, Revotskie N, Stokes J III. Factors of risk in the development of coronary heart disease - six year follow-up experience: the Framingham Study. Ann Intern Med 1961; 55:33-50.

10. Multiple Risk Factor Intervention Trial Research Group. Multiple Risk Factor Intervention Trial: risk factor changes and mortality results. JAMA 1982;248:1465-77. 11. Veterans Administration Cooperative Study Group on Antihypertensive Agents. Effects of treatment on morbidity in hypertension: results in patients with diastolic blood pressures averaging 115 through 129 mm Hg. JAMA 1967;202:1028-34.

12. Veterans Administration Cooperative
Study Group on Antihypertensive Agents. Effects of treatment on morbidity in hypertension. II. Results in patients with diastolic blood pressure averaging 90 through 114 mm Hg. JAMA 1970;213:1143-52.

13. Jones DW, Hall JE. The National High Blood Pressure Education Program: thirty years and counting. Hypertension 2002; 39:941-2.

14. Hypertension Detection and Followup Program Cooperative Group. Five-year findings of the hypertension detection and follow-up program. I. Reduction in mortality of persons with high blood pressure, including mild hypertension. JAMA 1979;242:2562-71.

15. The Australian Therapeutic Trial in mild hypertension: report by the Management Committee. Lancet 1980;1:1261-7. 16. Amery A, Birkenhäger W, Brixko $\mathrm{P}$, et al. Mortality and morbidity results from the European Working Party on High Blood Pressure in the Elderly trial. Lancet 1985;1:1349-54.

17. Medical Research Council Working 
Party. MRC trial of treatment of mild hypertension: principal results. Br Med J (Clin Res Ed) 1985;291:97-104.

18. SHEP Cooperative Research Group. Prevention of stroke by antihypertensive drug treatment in older persons with isolated systolic hypertension: final results of the Systolic Hypertension in the Elderly Program (SHEP). JAMA 1991;265:3255-64. 19. Dahlöf B, Lindholm LH, Hansson L, Scherstén B, Ekbom T, Wester PO. Morbidity and mortality in the Swedish Trial in Old Patients with Hypertension (STOPHypertension). Lancet 1991;338:1281-5. 20. Staessen JA, Fagard R, Thijs L, et al. Randomised double-blind comparison of placebo and active treatment for older patients with isolated systolic hypertension. Lancet 1997;350:757-64.

21. Hansson L, Zanchetti A, Carruthers SG, et al. Effects of intensive blood-pressure lowering and low-dose aspirin in patients with hypertension: principal results of the Hypertension Optimal Treatment (HOT) randomised trial. Lancet 1998;351: 1755-62.

22. The ALLHAT Collaborative Research Group. Major cardiovascular events in hypertensive patients randomized to doxazosin vs chlorthalidone: the antihypertensive and lipid-lowering treatment to prevent heart attack trial (ALLHAT). JAMA 2000;283:1967-75.
23. The ALLHAT Officers and Coordinators for the ALLHAT Collaborative Research Group. Major outcomes in highrisk hypertensive patients randomized to angiotensin-converting enzyme inhibitor or calcium channel blocker vs diuretic: the Antihypertensive and Lipid-Lowering Treatment to Prevent Heart Attack Trial (ALLHAT). JAMA 2002;288:2981-97.

24. Beckett NS, Peters R, Fletcher AE, et al. Treatment of hypertension in patients 80 years of age or older. N Engl J Med 2008; 358:1887-98.

25. The ACCORD Study Group. Effects of intensive blood-pressure control in type 2 diabetes mellitus. N Engl J Med 2010;362: 1575-85.

26. The SPRINT Research Group. A randomized trial of intensive versus standard blood-pressure control. N Engl J Med 2015; 373:2103-16.

27. Lonn EM, Bosch J, López-Jaramillo P, et al. Blood-pressure lowering in intermediate-risk persons without cardiovascular disease. N Engl J Med 2016;374:2009-20. 28. Chobanian AV, Bakris GL, Black HR, et al. The Seventh Report of the Joint National Committee on Prevention, Detection, Evaluation, and Treatment of High Blood Pressure: the JNC 7 report. JAMA 2003;289:2560-72.

29. Dahlöf B, Devereux RB, Kjeldsen SE, et al. Cardiovascular morbidity and mor- tality in the Losartan Intervention For Endpoint reduction in hypertension study (LIFE): a randomised trial against atenolol. Lancet 2002;359:995-1003.

30. Julius S, Kjeldsen SE, Weber M, et al. Outcomes in hypertensive patients at high cardiovascular risk treated with regimens based on valsartan or amlodipine: the VALUE randomised trial. Lancet 2004; 363:2022-31.

31. Jamerson K, Weber MA, Bakris GL, et al. Benazepril plus amlodipine or hydrochlorothiazide for hypertension in highrisk patients. N Engl J Med 2008;359:241728.

32. Blood Pressure Lowering Treatment Trialists' Collaboration. Effects of different blood-pressure-lowering regimens on major cardiovascular events: results of prospectively-designed overviews of randomised trials. Lancet 2003;362:1527-35.

33. The SPS3 Study Group. Blood-pressure targets in patients with recent lacunar stroke: the SPS3 randomised trial. Lancet 2013;382:507-15.

34. James PA, Oparil S, Carter BL, et al. 2014 Evidence-based guideline for the management of high blood pressure in adults: report from the panel members appointed to the Eighth Joint National Committee (JNC 8). JAMA 2014;311:50720.

Copyright @ 2016 Massachusetts Medical Society. endocrinology, genetics, infectious disease, nephrology, pediatrics, and many other medical specialties. These pages, along with collections of articles on clinical and nonclinical topics, offer links to interactive and multimedia content and feature recently published articles as well as material from the NEJM archive (1812-1989). 